\title{
Researching Agent Technologies in the Electronic Classroom
}

\author{
Carolyn Dowling \\ Australian Catholic University, 115 Victoria Parade, Fitzroy, Victoria 3065, Australia \\ c.dowling@patrick.acu.eduau
}

Keywords: research, innovation, lifelong learning/education, distance learning, computer assisted instruction

\begin{abstract}
Designers of online education are increasingly making use of the Internet not just to enable students to access information, but to provide opportunities for communication between the various participants in the learning process. These frequently include a range of more or less personified and 'intelligent' computer programs generally referred to as 'agents', many of which are designed to play a key role in the social interactions that mediate learning. Through discussion of a selection of current implementations of agent technology within educational contexts, this paper highlights some key directions for ongoing research and evaluation.
\end{abstract}

\section{INTRODUCTION}

An important consequence of the increased rate of technological and social change that is being experienced in most parts of the world is the formal recognition by government, industry and educational institutions, of the need for increased flexibility in the provision of opportunities for learning. Computer based learning environments, particularly those that are Internet based, are one obvious vehicle for achieving such flexibility. Online education is becoming an increasingly popular alternative to traditional face to face modes of teaching at all levels, from elementary education to tertiary institutions and on-the-job technical or professional training. Within these 
contexts the Internet not only enables students to access information but, in keeping with current emphases on the importance of social interaction in the construction of knowledge, it also provides opportunities for communication between the different participants in the learning process.

Within a number of online educational environments we are now witnessing the emergence of a new participant in the social interactions that mediate learning. The substitution of 'agents', computer programs possessed of varying degrees of intelligence, autonomy and 'personality', for different aspects of human presence in the classroom, raises a number of issues in relation to the processes through which knowledge is constructed, and the qualities which permit successful participation in those processes.

Software agents are already a familiar part of our computing experience, from the anonymous programs that scurry about the Internet locating information for search engines to the cheerful little characters that pop up on our screens offering assistance with tasks ranging from formatting a date to constructing a complex audio-visual presentation. We may even have interacted with software 'characters' in 'chat' environments without realising they were not in fact human.

An early but still useful conception of a software agent with which many educators would be familiar is, "A character, enacted by the computer, who acts on behalf of the user in a virtual environment", useful in mediating “... a relationship between the labyrinthine precision of computers and the fuzzy complexity of man" (Laurel 1990, p.355). The practical implementation of this concept has resulted in a range of more functional definitions such as, "An agent can be viewed as an object which has a goal and autonomously solves problems through interaction, such as collaboration, competition, negotiation and so on" (Kinoshita and Sugawara 1995).

Summarising the writings of a number of researchers, Aroyo and Kommers (1999, p.237) identify four major characteristics of agents as being autonomy, responsiveness or reactiveness, pro-activeness and social ability. Other qualities frequently proposed, but not supported by all researchers or indeed by all users, include the ability to learn from experience and consequently to respond in flexible and possibly unforeseen ways to particular situations, and the possession of a believable 'character' or personality as a basis for social interaction.

As is the case with agents in the physical world, there are many possible roles for software agents. They may represent the interests of individuals as would a trustworthy personal servant. They may be modelled on the personal qualities and preferences of a person, and so function as a type of electronic 'clone' within electronic environments where the user is not able to be present 'in person', or they might fulfil more complex roles in which they represent the interests of more than one person and act as 'brokers' of deals. 
They may present themselves to us openly, or function in the background, somewhat in the manner of a 'secret agent' in the physical world. According to the complexity and sophistication of their programming they may have a greater or lesser degree of independence of action, and a more or less de'veloped 'character'.

\section{AGENTS IN THE ELECTRONIC CLASSROOM}

Agents within educational contexts are designed to fulfil a range of purposes. Types of agents currently implemented in projects around the world include record keepers, information seekers, testers, facilitators of collaboration, tutors or instructors, fellow learners and tutees. Some operate behind the scenes entailing minimal interaction with students. These are of only marginal relevance to the main focus of this paper. Others either wholly or partly substitute for human-to-human interaction. In terms of our current understandings of the extent to which knowledge is socially constructed, this is for many people the most valued and pedagogically important aspect of the face to face classroom. Indeed it might be argued that unless it can be demonstrated that the social interactions that mediate face to face learning have a digital equivalent, online courses will inevitably be regarded by most educators as 'second best'.

The 'social' role of instructor or tutor has of course long been associated with educational uses of computers, albeit in forms now generally regarded poorly by most educators. Other socially interactive roles such as that of 'fellow learner' are more obviously innovative, and suggest the possibility of a far richer and more complex interactivity within electronic educational environments than that to which we are accustomed. Each role raises special challenges, not simply in relation to the technical capabilities of the software, but in terms of our understanding of the nature of both teaching and learning.

While research undertaken from an $\mathrm{AI}$ perspective necessarily focuses on the technical capabilities of agents, research within the special context of education needs to place greater emphasis on matching the capacities of the software with current understandings of pedagogical theory. How effectively does engagement with a software agent substitute for the various dimensions of human to human pedagogical interactions? Further, do such interactions incorporate the potential to extend our existing understandings of what constitutes an effective learning environment?

Johnson (1998) has proposed the following definition the role of a pedagogical agent: 
"Pedagogical agents are autonomous agents that support human learning, by interacting with students in the context of interactive learning environments. They extend and improve upon previous work on intelligent tutoring systems in a number of ways. They adapt their behaviour to the dynamic state of the learning environment, taking advantage of learning opportunities as they arise. They can support collaborative learning as well as individualised learning, because multiple students and agents can interact in a shared environment. Given a suitably rich user interface, pedagogical agents are capable of a wide spectrum of instructionally effective interactions with students, including multimodal dialog. Animated pedagogical agents can promote student motivation and engagement, and engender affective as well as cognitive responses" (Johnson 1998, p.13).

This is a comprehensive and optimistic vision, incorporating a number of possible roles for software agents within educational environments. Many of these have already been implemented in various contexts. In evaluating the success of existing implementations and in considering future directions, several issues stand out as necessitating further research in relation to their implications for learning.

\section{RESEARCH ISSUES}

One of the key issues for developers of software agents is the degree to which these programs should appear to the user to be 'human'. In the electronic classroom this question clearly relates more to the socially interactive agents than to those performing more tool-like functions, which arguably would require a less developed 'personality'. There are strong arguments that a degree of personification of computer interfaces is inevitable. As Shirk put it:

"Although there is some dispute among software critics concerning the advisability of having 'personalities' in computer programs, their presence seems unavoidable. Any time there is communication between a computer and a human, the information presented by the computer has a certain style, diction, and tone of voice which impact upon the human's attitude and response toward the software" (Shirk 1988, p.320).

Further, our current understandings of learning depend very much on an acceptance of the role of the social construction of knowledge. It is in this regard that online interactions of a 'social' nature with a significantly personified entity can be perceived as adding value to educational activities

However the degree to which personification should be deliberately fostered is not entirely clear. While we might intuitively assume that computer users would prefer a highly personified interface, some research 
suggests that the reality is less straightforward. For instance, users react badly if a software 'personality' represented on the screen by an extremely realistic video image is unable to support its appearance with an appropriately high level of knowledge and understanding. As Masterton, puts it, "A common problem with AI programs that interact with humans is that they must present themselves in a way that reflects their ability. Where there is a conflict between the ability of the system and the users' perception of that ability a breakdown occurs and users may either fail to exploit its full potential or become frustrated with its shortcomings" (Masterton 1998, p.215). He goes on to suggest the implementation of a degree of anthropomorphism intended to convey qualities such as friendliness and usefulness, without the implication of possession of full human capabilities (Masterton 1998, p.211).

Some developers have addressed this issue through creating 'characters' that are more sketchily represented, for instance through cartoon-like graphics. This is a delicate and complex matter however, as some users also react badly to being given instructions or correction by an entity which they would normally regard as inferior to them - such as the animated paper clip familiar to users of Microsoft Office. Within some existing electronic learning environments for young students, the problem is cleverly addressed through the use of agents presented in the guise of various types of animals. Examples of this are the 'learning companions' developed by Chan (Chan 1996, Chan 1998).

In educational contexts this is an extremely complex issue, in view of the nature of the relationship between knowledge and authority or power in the classroom setting. It can be complicated still further within electronic environments by the special expectations students of different cultures might have in regard to how they interact with teachers as well as with fellow learners. Further, it is likely that optimal degrees and styles of personification might differ in accordance with the ages of students. This is certainly an aspect of the implementation of pedagogical agents which require considerable research

Another important issue in the development of educational software agents is their degree of autonomy or independence of action. Autonomy in the context of agents, is generally understood as meaning that they have the ability to make certain 'decisions' in the course of undertaking an activity, without the need for constant monitoring and intervention. Implicit in the recognition of the need for a capacity to exercise initiative is an acknowledgement that some outcomes of agent activity may not be easily predictable. This could pose certain difficulties for both teachers and students. From an educational point of view, the strong tendency in contemporary thinking to give more, rather than less control to the learner, 
suggests that limits should perhaps be placed on the capacity of educational agents to act independently. While they may be programmed so as to exercise considerable control over the learner, they may also be configured so as to be more sensitive to individualised user models, and more responsive to instruction from the student. However, while in the latter case the agent would have a greater degree of responsibility towards the perceived needs and wishes of learner, this may be contrary to the pedagogical goals of the teacher. The balance of freedom and control inherent in the structures and processes of more traditional learning environments depends to a significant extent on the ability of human teachers to sensitively modify their roles in response to the immediate situation. The ability of agents to achieve this is necessarily limited by the range of possibilities envisaged by their programmers. This is another aspect of pedagogical agents requiring further exploration by researchers.

A further concern in regard to the autonomy of pedagogical agents relates to the issue of intervention in the learning process. Despite the finding of Aroyo and Commers $(1999$, p.237) that pro-activity is a quality frequently sought after in agents, there is an important issue of balance to be addressed in relation to the educational process. It is well accepted that a high degree of unsought assistance whether from a human teacher or an excessively diligent and proactive agent can be quite detrimental, in particular to the metacognitive aspects of learning. Of course this is also an issue for teachers and learners in face-to-face educational contexts!

Closely associated with our ambivalence towards agent autonomy is the issue of trust. Teachers are generally accepted as being among the most trusted members of our community, both in relation to their responsibilities towards their students and also in broader terms. How do we decide whether to invest similar 'trust' in a software agent? While to an extent we can judge the competency, even the 'good intentions' of an agent through the outcomes of its actions, if we have had no hand in its programming we have little insight into its capabilities and limitations, even into what we might describe as its 'motivations'. Even if we are prepared to trust the agents with whom we deal directly, a further question remains as to how agents might reasonably 'decide' how to trust one another. In a number of fields, agents already have the capacity to interact with other agents emanating from different systems, and the exploration of such possibilities is very much a part of some research agendas in education. This another area in which extensive dialogue between educators and researchers would seem to be desirable.

The whole notion of an agent as a participant in the social construction of knowledge raises issues regarding whether or not computer programs can be said to 'learn' in a sense equivalent to that of human beings. If not, can they 
usefully participate in this aspect of a learning environment? The belief that they can do so is central to the thinking of many theorists and developers. Sheremetov and Nunez (1999), for example, whose work derives overtly from the theoretical frameworks of Piaget and Vygotsky, argue that:

"The design of learning environments, virtual or not, aims to promote productive interactions. In this type of learning a student changes from being a passive information receiver to an active collaborator, interacting with the tutors and colleagues in the learning process. Learning does not only result from acquiring knowledge, solving problems or using tools, but also from interacting about these on-going activities with persons and agents" (Sheremetov and Nunez 1999, p.305-306).

In relation to their specific project they write: "Our emphasis lies in the role of interactions in an artificial learning community as a group of real and artificial learners, tutors, and facilitators, working, supporting and learning from each other" (Sheremetov and Nunez 1999, p.306).

On the other hand it has been argued quite extensively that even the most heavily personified and autonomous of computer programs suffer from an intrinsic lack of ability to participate in the metacognitive aspects of learning. Pufall (1988), for instance, expresses a strong belief that a computer program is unable at any level commensurate with human capacities to modify its own knowledge structures or cognitive processes, and so cannot be regarded as a co-constructor of knowledge in a meaningful sense. While this might well have been the case in relation to earlier computer based learning environments, can we continue to make the same claims with confidence today or in the future? The capacity of software to 'learn' and adapt to experience through the incorporation of new information, the appropriate modification of its representation of the context in which it functions (its 'world'), and of its inference mechanisms, is undoubtedly increasing. This suggests that, whatever the differences in the underlying learning mechanisms, it might be possible to program agents that are able to convince their human companions that they are 'fellow learners'. Whether this is sufficient to enhance the learning experiences of students is another area requiring investigation.

At the core of the implementation of agent technologies in any context is the assumption that the range of tasks undertaken by single human being in a particular role can be effectively enacted by a multiplicity of agents. These should ideally be capable of interacting not only with the user but also with one another, and increasingly with agents associated with other programs. Within the electronic classroom the individual purposes of such entities derive from theoretical analyses of the component tasks and activities that are included in the larger scale pedagogical interactions of human beings. As educators, and indeed as students, we may simultaneously enact a range of 
roles in the classroom. The apparently unitary activity of 'teaching' involves such elements as demonstrating, guiding, telling, questioning, explaining, testing, motivating, criticising - even learning! Once identified, the assumption is that these diverse functions can be separated out and ultimately enacted through different configurations of agents working in relationships ranging from collaboration to competition. In keeping with the 'social' roles enacted by many of these agents, a metaphor commonly employed in conceptualising their aggregation is that of a 'society'.

Strategies for combining the activities of multiple agents already occupy a significant part of the research agenda in this area, and must continue to do so. However, while on the one hand the variety of functions enacted within a multi-agent environment is a clear attempt to realise the type of rich user interface described by Johnson (1998), some educators and researchers question the assumptions underlying these practices, arguing that they are underpinned by a reductionist rather than a holistic understanding of the processes and relationships involved in teaching and learning. In separating out the different components of pedagogical interactions, are we enabling each part to be realised more effectively, or are we failing to acknowledge that the global act of human teaching may in fact be more than the sum of its component parts? This is a research issue of a broader dimension than those concerned with issues of implementation.

\section{CONCLUSION}

In considering both actual and potential implementations of agent technology within the electronic classroom, it is clear that there is a pressing need for research not simply into the means of achieving the desired functionality, but also into the more complex question of the extent to which software entities are able to substitute for the delicate and subtle network of interactions and relationships which constitutes the social dimension of faceto-face pedagogy. To the extent that this can be realised, there is a further issue which must be addressed, namely the possibility that these technologies might have the potential to extent our understanding of the role of social relationships and interactions in the learning process.

\section{REFERENCES}

Aroyo, L and Kommers, P. (1999) Preface - intelligent agents for educational computeraided systems. Journal of Interactive Learning Research, 10 (3/4), pp.235-243. 
Chan, T.-W. (1996) Learning companion systems, social learning systems, and the global social learning club. Journal of AI in Education 7 (2).

Chan, T.-W. (1998) The past, present, and future of educational agents. [www.apc.src.ncu.edu.tw/apc/ppt_chan.html] 3.2.2000.

Johnson, W. L. (1998) Pedagogical agents. In Proceedings of the Sixth International Conference on Computers in Education (ICCE '98), T.-W. Chan, A. Collins and J. Lin (eds.), Beijing, October. pp.13-22.

Kinoshita, T. and Sugawara, K. (1995) Agent oriented computing. Soft Research Center, Tokyo.

Laurel, B. (1990) Interface agents: metaphors with character. In The Art of Human-Computer Interface Design, B. Laurel (ed.), Addison-Wesley, Reading, Massachusetts, pp. 355-367.

Masterton, S. (1998) Computer support for leamers using intelligent educational agents: the way forward. In Proceedings of the Sixth International Conference on Computers in Education (ICCE '98), T.-W. Chan, A. Collins and J. Lin (eds.), Beijing, October, pp. 211219.

Pufall, P. (1988) Function in Piaget's system: some notes for constructors of microworlds. In Constructivism in the Computer Age, G. Forman and P. Pufall (eds.), Lawrence Erlbaum Associates, Hillsdale, New Jersey.

Sheremetov, L. and Nunez, G. (1999) Multi-agent framework for virtual learning spaces. Journal of Interactive Learning Research, 10 (3/4), pp. 235-243.

Shirk, H. N. (1988) Technical writers as computer scientists: the challenges of online documentation. In Text, Context and Hypertext: writing with and for the computer, $\mathrm{E}$. Barrett (ed.), MIT Press, Cambridge, Massachusetts.

\section{BIOGRAPHY}

Carolyn Dowling is an Associate Professor and Head of the School of Arts and Sciences (Victoria) of Australian Catholic University. Her research interests include computer mediated writing, virtual reality in education and training contexts, social and ethical aspects of computing, software agents, and academic usage of the Internet. 